Article (refereed) - postprint

Hui, Cang; Richardson, David M.; Landi, Pietro; Minoarivelo, Henintsoa O.; Garnas, Jeff; Roy, Helen E. 2016. Defining invasiveness and invasibility in ecological networks. Biological Invasions, 18 (4). 971-983. 10.1007/s10530$\underline{016-1076-7}$

(C) Springer International Publishing Switzerland 2016

This version available http://nora.nerc.ac.uk/513373/

NERC has developed NORA to enable users to access research outputs wholly or partially funded by NERC. Copyright and other rights for material on this site are retained by the rights owners. Users should read the terms and conditions of use of this material at http://nora.nerc.ac.uk/policies.html\#access

This document is the author's final manuscript version of the journal article, incorporating any revisions agreed during the peer review process. There may be differences between this and the publisher's version. You are advised to consult the publisher's version if you wish to cite from this article.

The final publication is available at Springer via http://dx.doi.org/10.1007/s10530-016-1076-7 


\section{Defining invasiveness and invasibility in ecological networks}

2

3 Cang Hui ${ }^{1,2, *}$,

4 David M. Richardson ${ }^{3}$,

$5 \quad$ Pietro Landi ${ }^{1,4}$,

6 Henintsoa O. Minoarivelo ${ }^{1}$,

7 Jeff Garnas ${ }^{5}$,

8 Helen E. Roy ${ }^{6}$

9

10

11 Matieland 7602, South Africa; Africa; Matieland 7602, South Africa;

${ }^{1}$ Centre for Invasion Biology, Department of Mathematical Sciences, Stellenbosch University,

${ }^{2}$ BioMath Group, African Institute for Mathematical Sciences, Cape Town 7652, South

${ }^{3}$ Centre for Invasion Biology, Department of Botany and Zoology, Stellenbosch University,

${ }^{4}$ Evolution and Ecology Program, International Institute for Applied Systems Analysis, Schloßplatz 1, 2361 Laxenburg, Austria;

${ }^{5}$ Department of Zoology and Entomology, Forestry and Agricultural Biotechnology Institute, University of Pretoria, Pretoria 0002, South Africa;

${ }^{6}$ Centre for Ecology \& Hydrology, Wallingford, Oxfordshire, OX10 8BB, UK

*Correspondence: E-mail: chui@sun.ac.za 


\section{Abstract}

The success and failure of a biological invasion is context based, and yet two key concepts the invasiveness of species and the invasibility of recipient ecosystems - are often defined and considered separately. We propose a framework that can elucidate the complex relationship between invasibility and invasiveness. It is based on trait-mediated interactions between species and depicts the response of an ecological network to the intrusion of an alien species drawing on the concept of community saturation. Here, invasiveness of an introduced species with a particular trait is measured by its per capita population growth rate when the initial propagule size of the introduced species is very small. The invasibility of the recipient habitat or ecosystem is dependent on the structure of resident ecological network and is defined as the total width of opportunity niche in the trait space susceptible to invasion. Invasibility is thus a measure of network instability. We also correlate invasibility with the asymptotic instability of resident ecological network, measured by the leading eigenvalue of the interaction matrix that depicts trait-based interaction intensity multiplied by encounter rate (a pairwise product of propagule sizes of all members in a community). We further examine the relationship between invasibility and network architecture, including network connectance,

Keywords: biological invasions, fluctuating resource hypothesis, invasiveness, invasibility, ecological networks, interaction matrix, network stability, interaction strength 


\section{Introduction}

The search for generality in invasion ecology has progressed largely through quantifying the drivers behind two concepts separately and in concert: the invasiveness of alien species and the invasibility of recipient ecosystems (Richardson and Pyšek 2006). The concept of invasiveness follows a species-centric view of the introduction-naturalisation-invasion continuum (Blackburn et al. 2011). Much of the study of invasiveness has involved the identification and exploration of traits, barriers and drivers that determine location on this continuum for a given taxon (Richardson and Pyšek 2012). Invasiveness, or the propensity of invasive alien species (hereafter IAS) to invade, can be identified from comparative metrics between invasive and non-invasive alien species, such as those related to translocation bias, propagule pressure, and foraging/reproduction/dispersal traits (Pyšek and Richardson 2007). Invasiveness is further related to the potential impacts of IAS on the function and service of recipient ecosystems and thus dictates the prioritisation, prevention and control strategies in response to biological invasions (Blackburn et al. 2014). Of particular importance are the suite of traits of IAS that differ from those of native species and non-invasive alien species. A trait-based priority list of potentially highly invasive species can then be developed (Peacock and Worner 2008; Moravcová et al. 2015).

The second concept - invasibility - is a property of recipient ecosystems and involves the elucidation of features that determine its vulnerability to invasion such a community diversity, composition and assembly (Lonsdale 1999). Community assembly rules outline how species are "packed" in a community and how community composition is related to the occupied and available niche space in a given community. Early niche theories gave special attention to the role of biotic interactions in structuring communities (Tilman 2004). More recently, recognition that species assemblages in unsaturated local communities are at least in part driven by neutral forcing via the continuous influx of regional and alien species (Hubbell 2001; Stohlgren et al. 2003). Despite contrasting opinions on the applicability of neutral theory to real world communities (Chase 2005; Clark 2012; Rosindell et al. 2012), it is now widely accepted that both deterministic and stochastic processes interact to structure species assemblages (Bar-Massada et al. 2014; Nuwagaba et al. 2015).

To further elucidate the concept of invasibility, it is essential to first determine how an ecosystem responds to perturbations such as biological invasions, an issue that has been debated at least since May's (1974) proposition that complexity begets instability (McCann 2000; Fridley 2011, Allesina and Tang 2012). As the recipient ecosystem often comprises 
many interacting species, an ecological network provides an effective model for exploring the inherent complexity. A key aspect of this debate thus relates to connecting the different perspectives of network architecture to the stability of ecological networks. The architecture of an ecological network can be measured as particular features of the interaction matrix, depicting whether and how strongly two nodes interact. Typical features include connectance (the proportion of realized interactions among all possible ones; Olesen and Jordano 2002), nestedness (specialists only interacting with a subset of species with which generalists interact; Bascompte et al. 2003) and compartmentalization (a network can be grouped into delimited modules, measured by the level of modularity, where species are strongly interacting with species within the same module but not those from other modules; Newman 2006). Network complexity normally refers to a combined factor of network size (the number of nodes) and connectance (May 1974; Allesina and Tang 2012). Although consensus on the structure of mutualistic networks has been reached (e.g. Bascompte et al. 2003; Olesen et al. 2007; Guimarães et al. 2007; Thébault and Fontaine 2010; Mello et al. 2011), there is still considerable debate with respect to antagonistic networks (e.g., on whether antagonistic networks are more compartmentalised than random expectation; e.g. Poisot 2013).

Network stability, in contrast to network architecture, concerns how networks respond to perturbations (Yodzis 1981) and can be measured using different approaches (i.e., Lyapunov asymptotic stability, resilience, persistence and robustness, among others; May 1974; Pimm and Lawton 1978; Dunne et al. 2002; Donohue et al. 2013). The analysis of Lyapunov stability is a long established mathematical tool in dynamical systems for depicting whether a complex system will return to its local equilibrium after weak perturbations; this is typically assessed as the leading eigenvalue of the interaction matrix (May 1974; Allesina and Tang 2012). Such perturbations in an ecological network are often manifested as changes in population sizes caused by stochasticity or changing resources (Davis et al. 2000). Recent progress in resolving the complexity-stability debate has involved exploring the causal relationship between the architecture and stability of many mutualistic (e.g., plant-frugivore and plant-pollinator), trophic (food web) and antagonistic (predator-prey and hostparasite/pathogen) networks (e.g. Memmott et al. 2004; Eklof and Ebenman 2006; Bascompte et al. 2006; Burgos et al. 2007; Estrada 2007; Bastola et al. 2009; Kiers et al. 2010; Thébault and Fontaine 2010; Brose 2011; de Visser et al. 2011; Stouffer and Bascompte 2011; James et al. 2012), and explaining emergent network structures using dynamic network models with adaptive and random species rewiring (van Baalen et al. 2001; Kondoh 2003; Rezende et al. 2007; Vacher et al. 2008; Valdovinos et al. 2010; Zhang et al. 2011; Suweis et al. 2013; Minoarivelo et al. 2014; Nuwagaba et al. 2015). 
Here, we introduce invasibility as a new aspect of network instability. Full comprehension of the proposed framework of invasiveness and invasibility in ecological networks requires us first to establish the concept of community saturation in a network. This concept was initially developed from the theory on competition and limiting similarity (MacArthur 1972; Abrams 1983) where strong interspecific interactions preclude the establishment of IAS. A saturated ecological network can be defined as a particular community assemblage (a suite of species with their particular traits and population sizes) that cannot be invaded by an alien species given low propagule number, irrespective of that species' life history or relevant traits. Certainly, when the propagule size is too large, system behaviour will be overridden by the influx of propagules, making the concept of invasibility irrelevant. Very few, if any, ecological networks are truly saturated, as local communities can be strongly affected by regional species dynamics and stochasticity (Abrams 1998; Loreau 2000); rather the concept of community saturation serves as a theoretical benchmark by which invasibility can be measured. Specifically, the deviation from a saturated community can be measured by the cumulative niche space that permits invasion for a given recipient ecosystem.

Invasibility and invasiveness are not isolated concepts but are strongly interwoven. As an example, as Darwin (1859) first posited in what has become known as his "naturalization hypothesis", introduced species should be more successful (i.e., more invasive) when the recipient community lacks congeneric or ecologically similar species (Duncan and Williams 2002). The underlying logic of this statement relies on the assumption that closely related species show greater life history, trait and therefore niche overlap such that an intact community would be minimally invadable to congeners relative to more distantly related species. A counter-argument which has received some empirical support is that trait similarity among related species might predict habitat suitability and result in higher invasibility when congeners are considered (Duncan and Williams 2002). Either way, it is clear that the two core concepts - invasibility and invasiveness - are context dependent and closely related. Moreover, introduced species can often only invade certain native ecosystems following some form of perturbation (Davis et al. 2000; Shea and Chesson 2002). Here, we attempt to explicitly bridge these two concepts through trait-mediated interactions in ecological networks by visualising both in a single invasion fitness diagram. Since this is a new aspect of network instability, we also examine how invasibility is related to other network stability measures (specifically asymptotic stability), and how it is related to typical network structure. We exemplify this framework using a trait-mediated assembly-level model and discuss how invasibility can be practically assessed with poor data quality by using qualitative interaction matrix, in cases for selected invasive alien insects in a variety of ecosystems. 


\section{Invasion fitness}

160

Assembly-level models have a long history in community ecology (e.g. Drake 1990; Morton and Law 1997) and they normally assume infrequent colonization of new species from a predetermined regional species pool. Some recent assembly-level models further allow limited evolutionary processes (e.g. Drossel et al. 2001; McKane 2004) and adaptive response to disturbance (Kondoh 2003; Zhang et al. 2011; Suweis et al. 2013; Nuwagaba et al. 2015; Minoarivelo and Hui 2015; Hui et al. 2015). In particular, the model proposed by Loeuille and Loreau (2005) can depict the emergence of complex food webs through ecological and evolutionary processes involving trait-mediated interactions. Here, we use a food-web model with trait-mediated interactions to demonstrate the framework of invasiveness and invasibility. Specifically, we consider a generalised version of the Loeuille and Loreau (2005) model developed by Brännström et al. (2011) which depicts the per capita population change rate as a function of population growth derived from prey consumption minus mortality from senescence, predation and interference competition (Appendix A). This model is applied because assembly-level data on functional traits, population sizes and interaction strengths are often lacking, which precludes the study of a full network. Simply, targeting functional traits that appear especially important to the population demography of focal species (i.e., that affect the strength of density dependence or influence biotic interactions) makes model parameterization more tractable. Certainly, the absence of such data hampers the clarity when introducing the framework of network invasibility. For this reason, in what follows we will not discuss details of the model itself. Rather, we focus on introducing the framework with this model only serving as a tool for generating required data. In the absence of comprehensive knowledge of communities and community interactions, all is not lost - we discuss the protocol for cases with insufficient data later. As we shall see, even only characterizing the directionality of interactions among species without estimates of interaction strength can be effective in quantifying invasiveness and invasibility (Rossberg et al. 2010).

Generating a resident network of multiple heterotrophic species as depicted in the model (Appendix A) can be accomplished in two ways. First, many studies have followed a simple procedure of randomly assigning trait values and parameters for all initial species, running the model until equilibrium is reached, and then removing those species with population sizes below a certain threshold (Holland and DeAngelis 2010). At this stage the network is considered to be at its equilibrium. Once the recipient community has reached its equilibrium, we could consider the invasiveness of a potential introduced species as its 
invasion fitness, defined as its per-capita population growth rate when propagule size is trivial (close to zero) and the community is at equilibrium (Fig.1a). Invasion fitness is a good proxy of invasiveness for an introduced species - if the trait of an introduced species lies within the green intervals along the zero invasion fitness line (Fig.1a), the introduced species will experience positive invasion fitness and thus be able to establish and invade the resident community. If trait values land within the yellow intervals, the species will experience negative invasion fitness and thus be repelled by the resident community (Fig.1a). Clearly, not all species can invade the resident network (Fig.1a).

For a given introduced species with a particular trait, if there is a native resident species having an identical/similar trait (i.e. the trait of introduced species is close to any one red dot [traits of resident species] in Fig.1a), the invasion fitness will then become close to zero. Because of the zero population growth, such species are less likely to establish simply due to demographic stochasticity (the case of neutral coexistence). Even if these species establish they will not become invasive but persist at low abundance until either eliminated via ecological drift or increasing opportunistically in response to disturbance. If the trait of an alien species is quite different from those of any resident species (i.e. sitting between red dots in Fig.1a), it is then likely to become either highly invasive (peaks in green zones) or be quickly expelled from the network (valleys in yellow zones), with a 50/50 chance for successful invasion in a species-rich network due to the constraints on any dynamic systems given the continuity of the invasion fitness function (from the Fundamental Theorem of Algebra and the Central Limit Theorem). To this end, the invasibility of the recipient ecological network can be defined as the total width of opportunity niche in the trait space (i.e. the summation of all the green intervals).

As an alternative to the static trait approach taken above we could also generate a model community as an adaptive network, where species within the network can co-evolve according to adaptive dynamics (Brännström et al. 2011), or where species with different traits can be continuously introduced into the community from a large species pool (i.e. a meta-community; Gilpin and Hanski 1991; Hubbell 2001). This approach will potentially, but not always, lead to a saturated ecological network (Fig.1b). No alien species can invade a saturated network as the invasion fitness of any introduced species is equal to or less than zero (Fig.1b). These two ways of generating community assemblages sitting either at the equilibrium of ecological dynamics (Fig.1a) or the saturated assembly (Fig.1b) provide an update to the standard naturalisation hypothesis (Duncan and Williams, 2002). Of course, even if the saturated assembly does exist, a community under constant bombardment of IAS 
intrusion is not likely to be either on the equilibrium or remaining saturated but somewhere between the two extremes. Consequently, we examine how temporal and trait perturbation creates opportunity niche in ecological networks.

\section{Network invasibility under temporal perturbation}

Many factors can drive the change and cyclic fluctuation of resources including land use change, alteration of fire regimes, seasonality and weather conditions. In what follows, we introduce temporal perturbation to a saturated ecological network. Specifically, we examine the fluctuating resource hypothesis (Davis et al. 2000), where fluctuation in resource availability has been identified as the key factor mediating the susceptibility of an ecosystem to invasion by non-resident species. We set a cyclic dynamics to the primary producers (autotrophs in the model; Appendix A) using a sine function to modify resource levels with a particular pulse and magnitude and record the temporal invasion fitness caused by such periodic perturbation in the saturated ecological network.

Results confirmed that fluctuating resources can create an opportunity niche with positive invasion fitness when resource levels exceed original levels (Fig.2). The temporal invasiveness of an introduced alien species will reach the maximum when its trait value is optimal for consuming autotrophic resource. The invasibility (length of positive invasion fitness zone along trait axis in Fig.2) appeared high for the first half of the perturbation period (positive phase) and allowed a wide range of introduced species to invade (a long tail towards high trait/trophic direction) but quickly dropped to zero for the second half of the perturbation period (negative phase). This suggests that although perturbation might not create a persistent opportunity niche even for highly invasive alien species in a saturated community, environmental stochasticity can markedly increase the network invasibility in otherwise resistant assemblages.

Importantly, rapid resource increase (approaching the 1.0 perturbation period in Fig.2), created an opportunity niche for invasion especially for species with optimal traits for resource consumption, even though resource availability was still below the pre-perturbation levels. Similarly, rapid resource decline (happening at 0.5 perturbation period in Fig.2) also eliminated a portion of opportunity niche around the optimal trait. Evidently, the rate and direction of change in resources as well their absolute level can both affect ecological invasibility, with both playing similar roles in influencing network invasibility. This provides an interesting extension to the fluctuating resource hypothesis which posits that variability in resources promotes invasion in plants (Davis et al. 2000). It further echoes predictions of the 
paradox of enrichment (Rosenzweig 1971) where enhanced resource level can be accompanied by instability in a food chain.

\section{Network structure and stability}

To simulate an unsaturated ecological network, we randomly altered the trait of heterotrophic species in a saturated network, by an increment following a normal distribution with zero mean and a particular standard deviation (in this case, $\sigma=0.3$ ). Theoretically, we can then calculate the Jacobian for randomly-altered networks, with $a_{i j}=\partial\left(d n_{j} / d t\right) / \partial n_{i}$, defined at the population equilibriums. However, this is impossible in practice; instead, interaction strength is often measured as the observed rate of species $j$ interacting with species $i, a_{i j}=P_{i j} n_{i} n_{j}$, where $P_{i j}$ measures the per-capita interaction strength. Consequently, we calculated three network structures for 1000 randomly altered networks depicted by the matrix of observed interaction strengths. As these altered networks were generated from the same saturated assembly, they were of the same network size.

Metrics of network architecture are diverse. Essentially, these metrics descript different aspects of network structure based on the matrix of interaction strength. These metrics can be divided into two categories: those portraying the role of particular species in the network (e.g. centrality) and those portraying the structure of entire networks. As invasibility is related to the latter, we chose three metrics from this category that depict the three most important features of a matrix - its matrix sparsity, asymmetry and symmetry. In particular, these features are normally measured by three widely used metrics for quantitative networks: connectance, nestedness and modularity. The quantitative connectance metric was computed as the quantitative linkage density divided by the number of species in the network (Tylianakis et al. 2007). A highly connected network is formed largely by generalists with strong and evenly distributed interactions, whereas a less connected network is formed by specialists. We used the metric WINE (weighted interaction nestedness estimator) to quantify the level of nestedness (Galeano et al. 2009). Nested communities are often formed when both specialist and generalist species are present, and where specialists primarily interact with a subset of the partners of generalists. Finally, the level of modularity was measured using a new algorithm QuanBimo (Dormann and Strau $\beta$ 2014; adapted from Claused et al. 2008). A community with high modularity is compartmentalised into multiple species modules or motifs, with species within the same module interacting strongly with each other but not with species from other modules. All these network metric measurements were implemented in the R library bipartite v2.05 (Dormann et al. 2008). 
We examine how network metrics are associated with the invasibility (the total width of opportunity niche) and the maximum invasiveness (height of the peak invasion fitness). How the system is altered/deviated from its saturated assembly is depicted by the absolute deviation of traits from the traits of the saturated assembly (hereafter, distance to assembly saturation). The asymptotic instability of a system can be measured by the leading eigenvalue of its interaction matrix. When the leading eigenvalue is less than zero, the system will return to its local equilibrium after small perturbations; otherwise, the fluctuations in population abundance will be amplified.

As illustrated in Fig.3, there is a strong positive correlation between invasibility and maximum invasiveness (Pearson's $r=0.418, \mathrm{p}<0.001$ ), suggesting an invasive species often performing more invasively in highly invadable ecosystems. Invasibility showed a positive correlation to the distance to assembly saturation $(r=0.106, \mathrm{p}=0.0005)$. In contrast, invasibility showed a negative correlation to ecological stability measured by the lead eigenvalue $(r=-0.267, \mathrm{p}<0.0001)$. Evidently, since distance to assembly saturation and asymptotic stability reflect different aspects of interaction networks, they have different implications for understanding network function. Such an opposing relationship between the distance to assembly saturation and asymptotic stability is also evident in literature. For instance, Allesina and Tang (2012) reconfirmed that asymptotic stability is negatively affected by nestedness in bipartite mutualistic networks, and as such at ecological time scales, an ecosystem dominated by mutualistic interactions is likely unstable and species poor. In contrast, mutualistic communities can maximise structural stability through potentially enhanced nestedness (Rohr et al. 2014); that is, at long-term time scales, mutualistic interactions can act as a stabilizing force and restrict diversification (Raimundo et al. 2014). Invasibility also shows strong positive correlations $(\mathrm{p}<0.0001)$ with all three measurements of network architecture (with quantitative connectance, $r=0.266$; with nestedness, $r=0.179$; with modularity, $r=0.324)$. When invasibility is unknown, we could predict it from asymptotic stability and the three measurable network architectures. A generalized linear model of the generated data showed that connectance and modularity are two strong predictors, with predicted invasibility from only these two network structures showing a strong correlation with observed ones $(r=0.325, \mathrm{p}<0.0001)$.

Although these results only reflect non-causal correlations between network architectures and invasibility, we could still contemplate the following ecological explanations for these positive correlations. First, May (1974) devised a necessary condition, further generalised by Allesina and Tang (2012), to ensure the stability in a complex network, 
$\alpha(S C)^{1 / 2}<\beta$, where $\alpha$ stands for the standard deviation of interaction strength, $S$ species richness, $C$ connectance and $\beta$ self-regulating force (e.g. negative density dependence). It suggests that the stability of a network requires stronger self-regulating force (large $\beta$ ) than potential reinforcing feedbacks from interspecific interactions, captured by the left side of the inequality. A highly connected network (large $C$ ) could encompass more reinforcing feedbacks between species, violating this condition and thus rendering network instability. Unstable networks, either due to reinforcing feedbacks or disturbance, could create opportunity niches for invasion, thus augmenting invasibility. Second, a highly nested network suggests a strong hierarchy and asymmetry, potentially from sorting species through multiple ecological filters, with the most extreme specialists only interacting with the most extreme generalists. This asymmetry could potentially create unbalanced energy/material flow from specialists to generalists, creating opportunity niches for introduced specialists that can exploit, perhaps more efficiently than resident generalists, the resident specialists. That is, network asymmetry creates opportunity niches for specialists and thus enhances invasibility. However, as the correlation between nestedness and invasibility is weak, further investigations are needed, especially for different types of ecological networks. Finally, a highly compartmentalized network is formed by clearly bounded modules, with species between modules rarely interacting. This suggests that these modules could have spatially or temporally partitioned available niches and habitats. This nevertheless provides opportunities for the invaders that can explore two or more modules. Species possessing traits with high plasticity or tolerance, and those with complex life cycles (through ontogenetic niche shift), could invade highly compartmentalized networks.

\section{Invasibility assessment}

The above demonstration with the trait-based assembly model suggests that to be capable of directly assessing the invasibility of a recipient ecosystem we need to rely on a comprehensive picture of functional traits that affect biotic interaction strengths and thus population dynamics, as well as overall densities. In practice, however, sufficient data are rarely available for estimating invasibility directly, even when global monitoring and webbased data sharing are actively seeking to make full records of species densities and traits in prioritised ecosystems available. For a rapid assessment of invasibility, we need an indirect or alternative way of capturing the interaction matrix. As shown above, an effective and conceptually and logistically tractable approach is to construct the interaction matrix of the recipient ecosystem (Fig.4a, top). In the absence of a quantitative matrix, a qualitative matrix 
would suffice. Conservation agencies could work with local experts to compile a semiquantitative interaction matrix (Fig.4a, middle) with weak interactions indicated by strength 0.1 , intermediate interactions by 1 , and strong interactions by 10 . In cases where data were even scarcer, a binary interaction matrix can be used (Fig.4a, bottom). While accurate interaction strengths make enhance predictive power, qualitative matrices are largely sufficient to understand network behaviours. That said, incorrect designation of the directionality of interactions $(+,-$ or 0$)$ may strongly bias the assessment of stability (Quirk and Ruppert, 1965; May, 1973; Jeffries, 1974). Ideally, the matrix should reflect the full species list of the recipient ecosystem. This can also be relaxed by only considering the potential networks that an introduced species will likely to impact or interact with, referred to here as an invader-centric ecological network.

Using an estimated interaction matrix at three different levels of acuity (quantitative, semi-quantitative and binary) we infer and compare invasibility from network architecture we use the well-studied biocontrol agent cum invader, the Harlequin ladybird, Harmonia axyridis (Pallas) (Coleoptera: Coccinellidae) which is predicted to be a major threat to other species within the aphidophagous guild (Roy et al. 2016). Native to Asia, H. axyridis has been introduced to many countries around the world as a biocontrol agent of aphids but it has spread to countries in which it was not intentionally introduced. It is now widespread and abundant in many regions and many habitats (Roy et al. 2016). Here, we compare the invasibility of two recipient habitats (agricultural and boreal systems in Europe; see Fig. 4b) to the invasion of $H$. axyridis. Many studies have demonstrated the potential interactions between $H$. axyridis and other aphidophagous species through laboratory and, to a lesser extent, field studies (Pell et al. 2008; Hautier et al. 2011; Brown et al. 2015; Roy et al. 2016). Harmonia axyridis engages in intraguild interactions with many species, including other aphid predators such as other ladybirds (Ware et al. 2009), lacewings (Santi and Maini 2006), and hoverflies (Ingels et al. 2015), as well as aphid parasitoids (Chacón et al. 2008) and aphid-pathogenic fungi (Roy et al. 2008). In general these interactions favour $H$. axyridis, though interaction strength and even direction can be influenced by the life stage of the interacting species (Felix and Soares 2004) and the environment (Gardiner et al. 2009).

Based on literature and expert opinions, we compiled the semi-quantitative interaction matrices of the European agricultural and boreal systems that are currently being invaded by $H$. axyridis (Fig. 4c and d). The lead eigenvalue before the invasion (removing the entries related to $H$. axyridis in the matrix) is effectively zero for both the agricultural system and boreal forests (absolute value less than $10^{-17}$ ), suggesting that both systems are at weak 
ecological equilibriums (asymptotically stable). After invasion by $H$. axyridis, both systems become ecologically unstable, with the boreal forests more unstable than the agricultural system (lead eigenvalue: 5.51 vs. 4.12), suggesting a stronger impact of $H$. axyridis on the boreal forests from the perspective of stability.

To calculate the three network structures, we replaced all negative entries with zeros as the calculation of these network metrics would otherwise report errors (note, for a predation interaction between species $i$ and $j$; if $a_{i j}>0$, we could either record $a_{j i}<0$ which is the convention or $a_{j i}=0$ which is the format for typical network analysis [used here]; Newman 2010). Prior to the invasion of $H$. axyridis in our estimated networks, the agricultural system has slightly higher connectance than the boreal system (0.38 vs. 0.34$)$, as well as low modularity $(<0.01)$ and a high level of nestedness $(>0.97)$. After the invasion, connectance dropped slightly in both habitats $(0.35$ vs. 0.32$)$ while modularity remained low $(<0.01)$ and the nestedness high $(\sim 1)$ in both.

Comparing these results with general expectations from our models, specifically the lead eigenvalue and connectance (Fig.3), the following interpretations can be proffered. First, before the invasion of $H$. axyridis, the agricultural system is more invadable than the boreal forest. As such, the invasion of $H$. axyridis would be more likely to happen first in the agricultural system. Second, after the invasion of $H$. axyridis, invasibility of both systems was reduced (reducing the risk of future invasion by similar invaders) although the agricultural system is still quite open for future invasions. The impact of the invasion of $H$. axyridis is more strongly felt by the boreal forest with its stability more disturbed (experiencing greater changes in the relative and absolute abundances of species after the arrival of $H$. axyridis). It is worth noting that the above exercise can be easily done before any invasions; a quick picture of the invasibility and the potential impact of the invasion quickly drawn and the invasibility between different habitats and ecosystems fairly compared. This can be accomplished using expert opinions, though the quality of the predictions is of course dependent on ecological realism in the interaction matrix.

\section{Conclusions}

We have demonstrated that ecological networks provide a good model for capturing the complexity of recipient ecosystems, and that the invasiveness of potential invaders and the invasibility of the recipient ecological networks can be defined using the concepts of invasion fitness and assembly saturation as a reference points. In this framework, invasiveness of a potential invader is defined as its invasion fitness (= per-capita population growth rate when 
the propagule size is trivial) and the invasibility of the network is defined as the width of opportunity niche available for potential invasions. This method is robust to the use of simplified interaction matrices for rapid assessments of network invasibility. Importantly, we argue that invasibility can be inferred from network stability, and that this is more closely linked to assembly saturation than ecological stability. The trait-based approach allowed for the testing of the fluctuating resource hypothesis, thus emphasizing its heuristic value.

\section{Acknowledgments}

This paper had its origin at a workshop on "Drivers, impacts, mechanisms and adaptation in insect invasions" hosted by the DST-NRF Centre of Excellence for Invasion Biology in Stellenbosch, South Africa, in November 2014. We are grateful to participants at this workshop and to Marc Kenis, Wolfgang Rabitsch, Michael Pocock, Ulf Dieckmann and Åke Brännström for discussions on the concepts presented in this paper. $\mathrm{CH}$ is supported by the South African Research Chair Initiative (SARChI), the National Research Foundation of South Africa (grant nos. 81825 and 76912), and the Australian Research Council (Discovery Project DP150103017). HOM receives a PhD Scholarship from the German Academic Exchange Service (DAAD). HER receives co-funding from the Natural Environment Research Council (NERC) and the Joint Nature Conservation Committee (JNCC). HER also acknowledges the COST Action TD1209. DMR acknowledges support from the National Research Foundation of South Africa (grant 85417).

\section{References}

Abrams PA (1983) The theory of limiting similarity. Annu Rev Ecol Syst 14:359-376

Abrams PA (1998) High competition with low similarity and low competition with high similarity: explorative and apparent competition in consumer-resource systems. Am Nat 152:114-128

Allesina S, Tang S (2012) Stability criteria for complex ecosystems. Nature 483:205-208

Bar-Massada A, Kent R, Carmel Y (2014) Environmental heterogeneity affects the location of modelled communities along the niche-neutrality continuum. Proc R Soc B 281:20133249

Bascompte J, Jordano P, Melián CJ, Olesen JM (2003) The nested assembly of plant-animal mutualistic networks. Proc Natl Acad Sci USA 100 (16):9383-9387 
Bascompte J, Jordano P, Olesen JM (2006) Asymmetric coevolutionary networks facilitate biodiversity maintenance. Science $312:$ 431-433

Bastola UM, Fortuna MA, Pascual-Garcia A, Ferrera A, Bascompte J (2009) The architecture of mutualistic networks minimizes competition and increases biodiversity. Nature 458:1018-1020

Blackburn TM, Essl F, Evans T, Hulme PE, Jeschke JM, Kühn I, Kumschick S, Marková Z, Mrugała A, Nentwig W, Pergl J, Pyšek P, Rabitsch W, Ricciardi A, Richardson DM, Sendek A, Vilà M, Wilson JRU, Winter M, Genovesi P, Bacher S (2014) A unified classification of alien species based on the magnitude of their environmental impacts. PLoS Biol 12:e1001850

Blackburn TM, Pyšek P, Bacher S, Carlton JT, Duncan RP, Jarošík V, Wilson JRU, Richardson DM (2011) A proposed unified framework for biological invasions. Trends Ecol Evol 26:333-339

Brännström A, Loeuille N, Loreau M, Dieckmann U (2011) Emergence and maintenance of biodiversity in an evolutionary food-web model. Theor Ecol 4:467-478

Brose U (2011). Extinctions in complex, size-structured communities. Basic Appl Ecol $12: 557-561$

Brown PMJ, Ingels B, Wheatley A, Rhule EL, De Clercq P, Van Leeuwen T, Thomas A (2015) Intraguild predation by Harmonia axyridis (Coleoptera: Coccinellidae) on native insects in Europe: molecular detection from field samples. Entomol Sci 18:130 133

Burgos E, Ceva H, Perazzo RPJ, Devoto M, Medan D, Zimmermann M, Delbue AM (2007) Why nestedness in mutualistic networks? J Theor Biol 249:307-313

Chacón J, Landis D, Heimpel G (2008) Potential for biotic interference of a classical biological control agent of the soybean aphid. Biol Control 46:216-225

Chase JM (2005) Towards a really unified theory for metacommunities. Funct Ecol 19:182186

Clark JS (2012) The coherence problem with the unified neutral theory of biodiversity. Trends Ecol Evol 27:198-202

Claused AM, Newman MEJ (2008) Hierarchical structure and the prediction of missing links in networks. Nature 453:98-101

Darwin CR (1859) On the origin of species. Murray, London 
Davis MA, Grime JP, Thompson K (2000) Fluctuating resources in plant communities: a general theory of invasibility. J Ecol 88:528-534

de Visser S, Freymann B, Olff H (2011) The Serengeti food web: empirical quantification and analysis of topological changes under increasing human impact. J Anim Ecol 80:465475

Donohue I et al. (2013). On the dimensionality of ecological stability. Ecol Lett 16:421-129

Dormann CF, Gruber B, Fruend J (2008) Introducing the bipartite package: Analysing ecological networks. R news 8:8-11

Dormann CF, Strau $\beta$ R (2014) A method for detecting modules in quantitative bipartite networks. Meth Ecol Evol 5:90-98

Drake JA (1990) The mechanics of community assembly and succession. J Theor Biol $147: 213-233$

Drossel B, Higgs P, McKane A (2001) The influence of predator-prey population dynamics on the long-term evolution of food web structure. J Theor Biol 208:91-107

Duncan RP, Williams PA (2002) Darwin's naturalisation hypothesis challenged. Nature 417:608-309

Dunne JA, Williams RJ, Martinez ND (2002) Network structure and biodiversity loss in food webs: robustness increases with connectance. Ecol Lett 5: 558-5657

Eklöf A, Ebenman B (2006) Species loss and secondary extinctions in simple and complex model communities. J Anim Ecol 75:239-246

Estrada E (2007) Topological structural classes of complex networks. Physical Review E 75:016103

Felix S, Soares AO (2004) Intraguild predation between the aphidophagous ladybird beetles Harmonia axyridis and Coccinella undecimpunctata (Coleoptera : Coccinellidae): the role of body weight. Eur J Entomol 101:237-242

Fridley JD (2011) Biodiversity as a bulwark against invasion: conceptual threads since Elton. In: Richardson DM (ed), Fifty years of invasion ecology: the legacy of Charles Elton, pp. 121-130 Wiley-Blackwell, Oxford

Galeano J, Pastor JM, Iriondo JM (2009) Weighted-interaction nestedness estimator (WINE): a new estimator to calculate over frequency matrices. Environ Model Softw 24:13421346 
Gardiner M, Landis D, Gratton C, DiFonzo C, O'Neal M, Chacon J, Wayo M, Schmidt N, Mueller E, Heimpel G (2009) Landscape diversity enhances biological control of an introduced crop pest in the north-central USA. Ecol Appl 19:143-154

Gilpin ME, Hanski IA (1991) Metapopulation dynamics: empirical and theoretical investigations. Academic Press, London

Guimarães PR et al. (2007) Build-up mechanisms determining the topology of mutualistic networks. J Theor Biol 249:181-189

Hautier L, San Martin G, Callier P, de Biseau JC, Grégoire JC (2011) Alkaloids provide evidence of intraguild predation on native coccinellids by Harmonia axyridis in the field. Biol Invas 13:1805-1814

Holland JN, DeAngelis DL (2010) A consumer-resource approach to the density-dependent population dynamics of mutualism. Ecology 91:1286-1295

Hubbell SP (2001) The unified neutral theory of biodiversity and biogeography. Princeton University Press, Princeton

Hui C, Minoarivelo HO, Nuwagaba S, Ramanantoanina A (2015) Adaptive diversification in coevolutionary systems. In: Pontarotti P (ed.), Evolutionary Biology: Biodiversification from Genotype to Phenotype. Springer, Berlin, pp.167-186.

Ingels B, Van Hassel P, Van Leeuwen T, De Clercq P (2015) Feeding History Affects Intraguild Interactions between Harmonia axyridis (Coleoptera: Coccinellidae) and Episyrphus balteatus (Diptera: Syrphidae). PLoS One 10:e0128518

James A, Pitchford JW, Plank MJ (2012) Disentangling nestedness from models of ecological complexity. Nature 487:227-230

Jeffries C (1974) Qualitative stability and digraphs in model ecosystems. Ecology 55:14151419

Kiers ET, Palmer TM, Ives AR, Bruno JF, Bronstein JL (2010). Mutualisms in a changing world: An evolutionary perspective. Ecol Lett 13:1459-1474

Kondoh M (2003) Foraging adaptation and the relationship between food-web complexity and stability. Science 299:1388-1391

Loeuille N, Loreau M (2005) Evolutionary emergence of size structured food webs. Proc Natl Acad Sci USA 102:5761-5766

Lonsdale WM (1999) Global patterns of plant invasions and the concept of invasibility. Ecology 80:1522-1536 
Loreau M (2000) Are communities saturated? On the relationship between $\alpha, \beta$ and $\gamma$ diversity. Ecol Lett 3:73-76

MacArthur RH (1972) Geographical ecology. Harper \& Row, New York

May RM (1974) Stability and complexity in model ecosystems. Princeton University Press, Princeton

McCann KS (2000) The diversity-stability debate. Nature 405:228-233

McKane AJ (2004) Evolving complex food webs. Eur Phys J B 38:287-295

Mello MAR et al. (2011) The modularity of seed dispersal: differences in structure and robustness between bat and bird-fruit networks. Oecologia 167:131-140

Memmott J, Waser NM, Price MV (2004) Tolerance of pollination networks to species extinctions. Proc R Soc B 271:2605-2611

Minoarivelo HO, Hui C (2015) Trait-mediated interaction leads to structural emergence in mutualistic networks. Evol Ecol, in press. DOI:10.1007/s10682-015-9798-z

Minoarivelo HO, Hui C, Terblanche JS, Kosakovsky Pond SL, Scheffler K (2014) Detecting phylogenetic signal in mutualistic interaction networks using a Markov process model. Oikos 123:1250-1260

Moravcová L, Pyšek P, Jarošík V, Pergl J (2015) Getting the right traits: reproductive and dispersal characteristics predict the invasiveness of herbaceous plant species. PLoS One 10:e0123634

Morton D, Law R (1997) Regional species pools and the assembly of local ecological communities. J Theor Biol 187:321-331

Newman MEJ (2006) Modularity and community structure in networks. Proc Natl Acad Sci USA 103:8577-8582

Newman MEJ (2010) Networks: an introduction. Oxford University Press, Oxford.

Nuwagaba S, Zhang F, Hui C (2015) A hybrid behavioural rule of adaptation and drift explains the emergent architecture of antagonistic networks. Proc R Soc B 282: 20150320

Olesen JM, Bascompte J, Dupont YL, Jordano P (2007) The modularity of pollination networks. Proc Nat Acad Sci 104:19891-19896

Olesen JM, Jordano P (2002) Geographic patterns in plant-pollinator mutualistic networks. Ecology 89:2416-2424 
Parker GA, Maynard Smith J (1990) Optimality theory in evolutionary biology. Nature $348: 27-33$

Peacock L, Worner SP (2008) Biological and ecological traits that assist establishment of alien invasive species. NZ Plant Prot 61:1-7

Pell JK, Baverstock J, Roy HE, Ware RL, Majerus MEN (2008) Intraguild predation involving Harmonia axyridis: a review of current knowledge and future perspectives. BioControl 53:147-168

Pimm SL, Lawton JH (1978) On feeding on more than one trophic level. Nature 275:542-544

Poisot T (2013) An a posteriori measure of network modularity. F1000 Research 2:130 (doi: 10.12688/f1000research.2-130.v3)

Pyšek P, Richardson DM (2007) Traits associated with invasiveness in alien plants: Where do we stand? In: Nentwig W (ed), Biological Invasions, pp. 97-125. Ecological Studies 193. Springer, Berlin

Quirk J, Ruppert R (1965) Qualitative economics and the stability of equilibrium. Rev Econ Stud 32:311-326

Raimundo RLG, Gibert JP, Hembry DH, Guimarães Jr PR (2014) Conflicting selection in the course of adaptive diversification: the interplay between mutualism and intraspecific competition. Am Nat 183:363-375

Rezende EL, Lavabre JE, Guimaraes Jr PR, Jordano P, Bascompte J (2007) Non-random coextinctions in phylogenetically structured mutualistic networks. Nature 448:925928

Richardson DM, Pyšek P (2006) Plant invasions - merging the concepts of species invasiveness and community invasibility. Prog Phys Geog 30:409-431

Richardson DM, Pyšek P (2012) Naturalization of introduced plants: ecological drivers of biogeographical patterns. New Phytol 196:383-396

Rohr RP, Saavedra S, Bascompte J (2014) On the structural stability of mutualistic systems. Science 345:1253497

Rosenzweig, M (1971) The paradox of enrichment. Science 171:385-387

Rosindell J, Hubbell SP, He F, Harmon LJ, Etienne RS (2012) The case for ecological neutral theory. Trends Ecol Evol 27:203-208 
Rossberg AG, Brännström, Å, Dieckmann, U. (2010) How trophic interaction strength depends on traits: a conceptual framework for representing multidimensional trophic niche spaces. Theor Ecol 3:13-24

Roy HE et al. (2016) Harmonia axyridis: an inspiration for global collaborations on invasion biology. Biol Invasions (in press)

Roy HE, Baverstock J, Ware R, Clark S, Majerus M, Baverstock K, Pell J (2008) Intraguild predation of the aphid pathogenic fungus Pandora neoaphidis by the invasive coccinellid Harmonia axyridis. Ecol Entomol 33:175-182

Santi F, Maini S (2006) Predation upon Adalia bipunctata and Harmonia axyridis eggs by Chrysoperla carnea larvae and Orius laevigatus adults. Bull Insectol 59:53-58

Shea K, Chesson P (2002) Community ecology theory as a framework for biological invasions. Trends Ecol Evol 17:170-176

Stohlgren TJ, Barnett DT, Kartesz J (2003). The rich get richer: patterns of plant invasions in the United States. Front Ecol Environ 1:11-14

Stouffer DB, Bascompte J (2011) Compartmentalisation increases food-web persistence. Proc Natl Acad Sci USA 108: 3648-3652

Suweis S, Simini F, Banavar JR, Maritan A (2013) Emergence of structural and dynamical properties of ecological mutualistic networks. Nature 500:449-452

Thébault E, Fontaine C (2010) Stability of ecological communities and the architecture of mutualistic and trophic networks. Sciences 329:853-856

Tilman D (2004) Niche tradeoffs, neutrality, and community structure: a stochastic theory of resource competition, invasion, and community assembly. Proc Natl Acad Sci USA 101:10854-10861

Tylianakis JM, Tscharntke T, Lewis OT (2007) Habitat modification alters the structure of tropical host-parasitoid food webs. Nature 445:202-205

Vacher C, Piou D, Desprez-Loustau M (2008) Architecture of an antagonistic tree/fungus network: the symmetric influence of past evolutionary history. PLoS One 3:e1740

Valdovinos FS, Ramos-Jiliberto R, Garay-Narvaèz L,Urbani P, Dunne JA (2010) Consequences of adaptive behaviour for the structure and dynamics of foodwebs. Ecol Lett 13:1546-1559

van Baalen M, Krivan V, van Rijn PCJ, Sabelis MW (2001) Alternative food, switching predators, and the persistence of predator-prey systems. Am Nat 157:512-524 
650 Ware RL, Yguel B, Majerus MEN (2009) Effects of competition, cannibalism and intra-guild predation on larval development of the European coccinellid Adalia bipunctata and the invasive species Harmonia axyridis. Ecol Entomol 34:12-19

653 Waxman D, Gavrilets S (2005) 20 questions on adaptive dynamics. J Evol Biol 18:1139-1154

654 Yodzis P (1981) The stability of real ecosystems. Nature 289:674-676

655 Zhang F, Hui C, Terblanche JS (2011) An interaction switch predicts the nested architecture 656 of mutualistic networks. Ecol Lett 14:797-803

657 
660 Fig.1: Invasion fitness of an introduced species as a function of its trait value relative to the

661

662

663

664

665

666

667

668

669

670

671

672

673

674

675

676

677

678

679

680

681

682

683

684

685

686

687

688

689

trait values of the resident species in the ecological networks. Red dots indicate the trait values of native resident species. (a) A randomly generated network at its ecological equilibrium. Only introduced species with traits lie in the green intervals can establish and invade the recipient ecological network; introduced species with traits lie in the yellow intervals will be repelled by the network (i.e. wiped out from the resident species/ecosystem). Invasibility is thus defined as the total width of the green intervals along the zero-fitness line. (b) A saturated assembly is defined as the recipient network with zero invasibility. Parameter values are the same as in Fig.1 of Brännström et al. (2011).

Fig.2: Invasion fitness of an introduced species as a function of its trait value in an ecological network. The resources of the saturated assembly in Fig.1b, $n_{0}$, are disturbed by adding a periodic perturbation (sine form with pulse 10 and magnitude 100). An alien species with the trait and timing of introduction in the positive invasion fitness zone can invade the ecological network; otherwise it will be repelled from the network. The peak of positive invasion fitness corresponds to the first quarter of the period and the optimal trait for resource consumption $(r$ $=\mu=2$ ).

Fig.3: Relationships between invasibility, maximum invasiveness, distance to assembly saturation (DAS) and the lead eigenvalue of the interaction matrix, as well as network architectures (quantitative connectance, levels of nestedness and modularity) in 1000 random ecological networks around the saturated assembly. Block tone corresponds to the frequency of networks.

Fig.4: Interaction matrices in practice. (a) An illustration of quantitative, semi-quantitative and qualitative interaction matrices (top, middle and bottom), with decreasing demands for data quality. (b) A picture of one European agricultural system with boreal habitat in background (Photo: H.E. Roy); both invaded by invasive alien ladybird Harmonia axyridis. Panels (c) and (d) are semi-quantitative interaction matrices for agricultural and boreal systems in Europe, based on literature and expert opinion. Acronyms: HA: Harmonia axyridis; AB: Adalia bipunctata; CS: Coccinella septempunctata; CC: Chrysoperla carnea; EB: Episyrphus balteatus; PN: Pandora neoaphidis; DC: Dinocampus coccinellae; AD: Adalia decempunctata; HS: Halyzia sedecimguttata; PF: Phorid fly; AP: aphids. 
Fig.1:

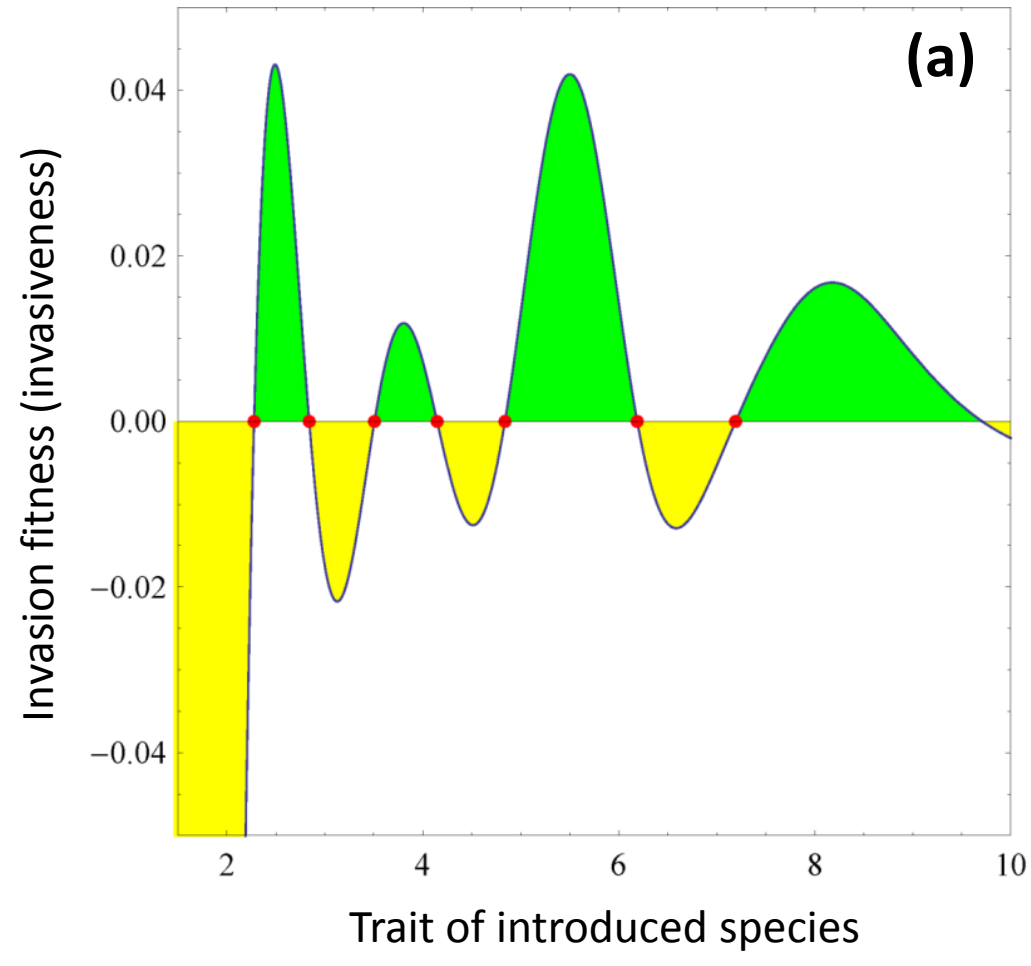

692

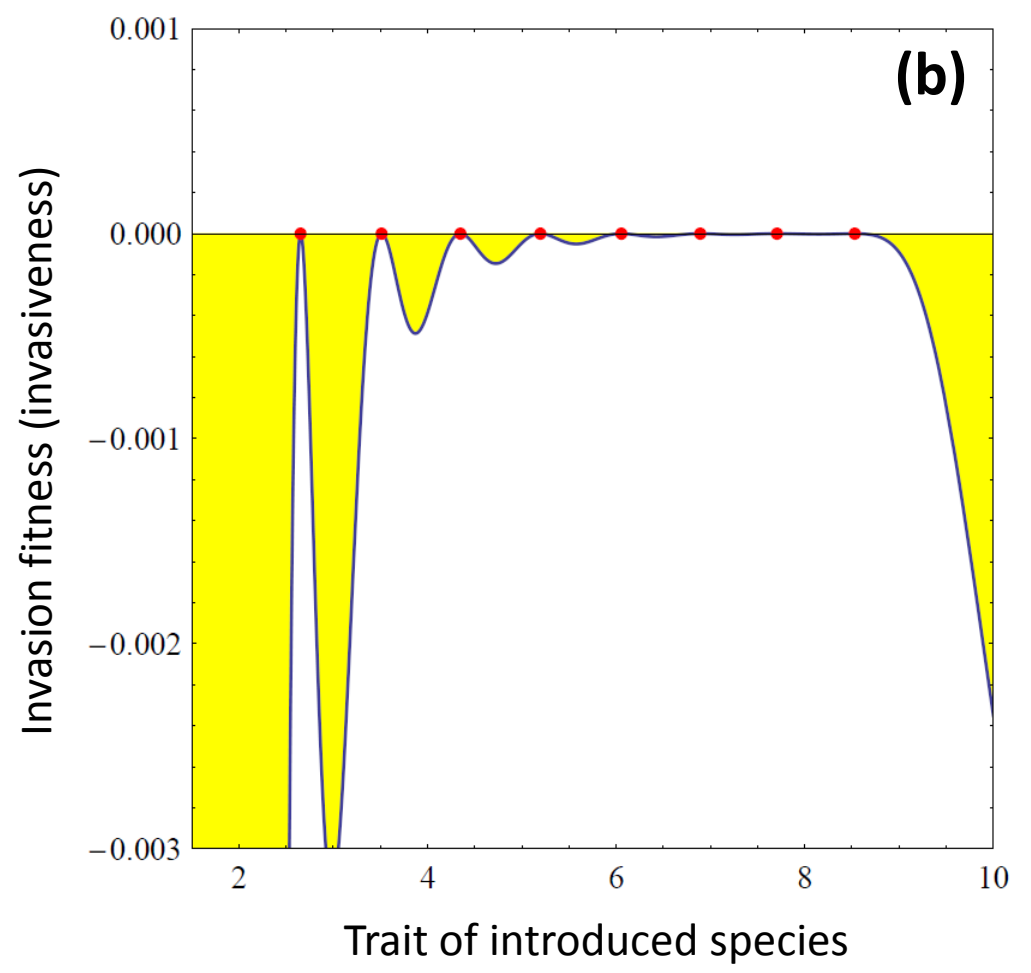

693

694

695

696 
697 Fig.2:

698

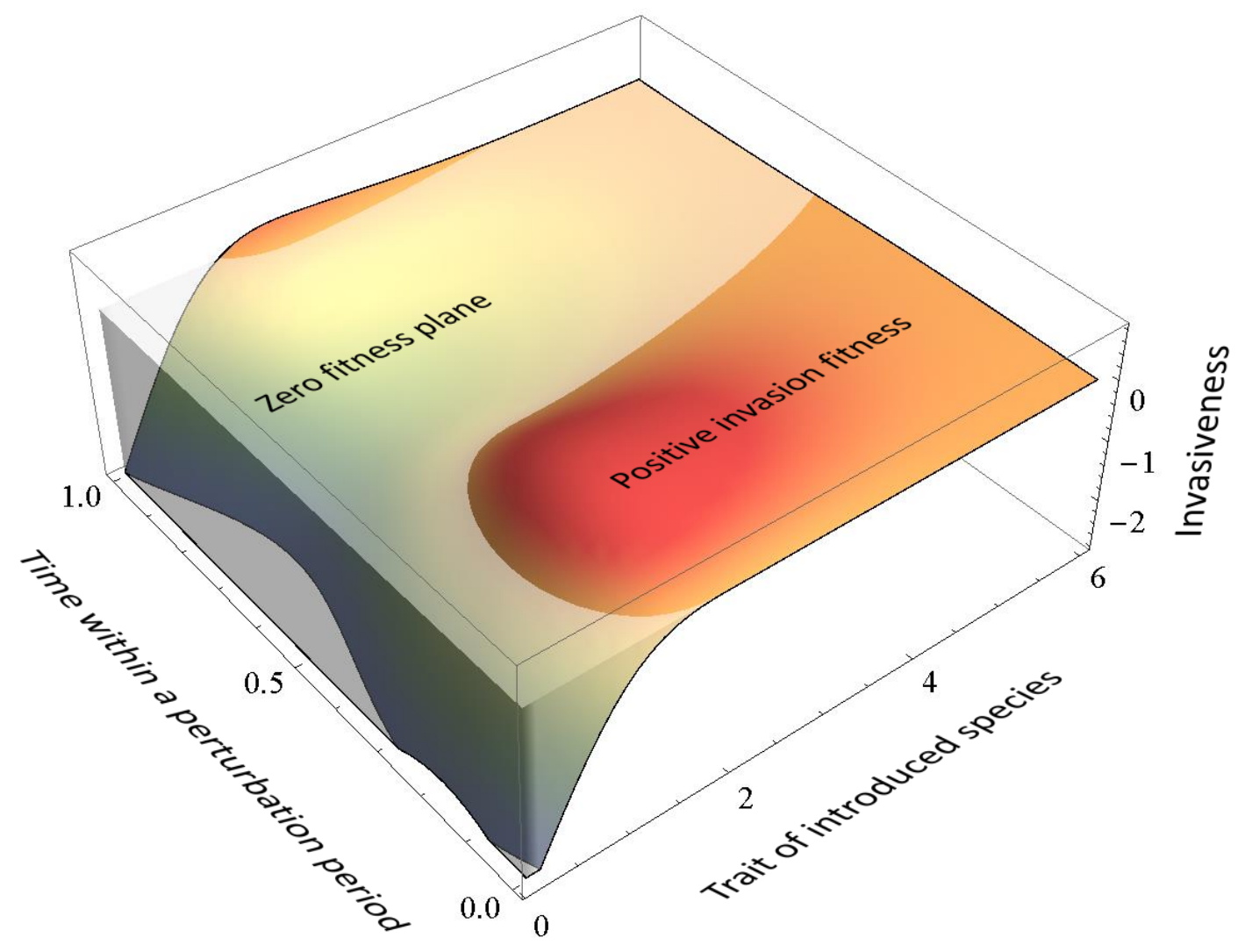


Fig.3:

700
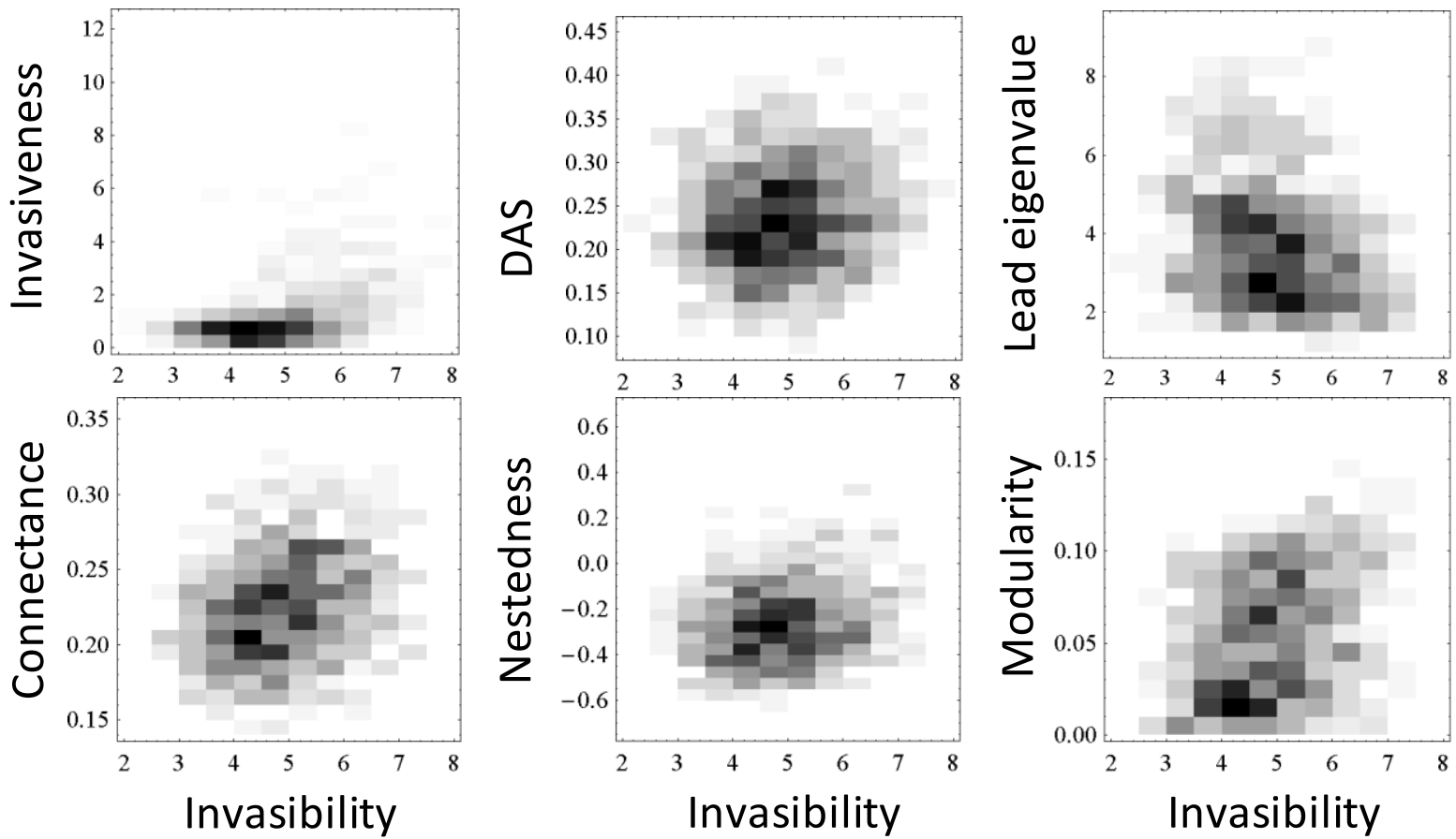

701 
Fig.4:

(a) \begin{tabular}{l|cccccc|} 
spp1 & -0.96 & -0.44 & -0.11 & 0.58 & -0.35 \\
spp2 & 0.33 & 0.58 & 0.65 & -0.13 & -0.85 \\
spp3 & -0.23 & -0.98 & -0.24 & -0.37 & 0.45 \\
spp4 & 0.64 & -0.44 & 0.36 & -0.01 & -0.43 \\
spp5 & -0.03 & -0.46 & 0.40 & -0.19 & 0.62 \\
\hline
\end{tabular}

\begin{tabular}{l|ccccc|}
\cline { 2 - 6 } spp1 & -10 & -1 & -0.1 & 1 & -1 \\
spp2 & 0.1 & 1 & 1 & -0.1 & -10 \\
spp3 & -0.1 & -10 & -0.1 & -1 & 1 \\
spp4 & 1 & -1 & 1 & -0.1 & -1 \\
spp5 & -0.1 & -0.1 & 1 & -0.1 & 1 \\
\cline { 2 - 6 }
\end{tabular}

\begin{tabular}{c|ccccc|}
\cline { 2 - 4 } spp1 & -1 & -1 & 0 & 1 & -1 \\
spp2 & 0 & 1 & 1 & 0 & -1 \\
spp3 & 0 & -1 & 0 & -1 & 1 \\
spp4 & 1 & -1 & 1 & 0 & -1 \\
spp5 & 0 & -1 & 1 & 0 & 1 \\
\cline { 2 - 6 } & & & & &
\end{tabular}

(c) $\mathrm{HA} \quad \mathrm{AB}$ CS CC EB PN DC AP

\begin{tabular}{l|rrrrrrrr|}
$\mathrm{HA}$ & -1 & 0 & -0.1 & -0.1 & 0 & 0 & -0.1 & 10 \\
$\mathrm{AB}$ & -10 & -0.1 & -0.1 & -0.1 & 0 & 0 & -0.1 & 10 \\
$\mathrm{CS}$ & -0.1 & 0 & -1 & -0.1 & 0 & 0 & -1 & 10 \\
$\mathrm{CC}$ & -10 & 0 & 0 & -0.1 & 0 & 0 & 0 & 10 \\
$\mathrm{~EB}$ & -10 & 0 & 0 & -0.1 & 0 & 0 & 0 & 10 \\
$\mathrm{PN}$ & 0.1 & 0.1 & 0.1 & 0 & 0 & 0 & 0 & 10 \\
$\mathrm{DC}$ & 0 & 0 & 0 & 0 & 0 & 0 & -0.1 & 0 \\
$\mathrm{AP}$ & -10 & -10 & -10 & -10 & -10 & -10 & 0 & 0 \\
\hline
\end{tabular}

(b)

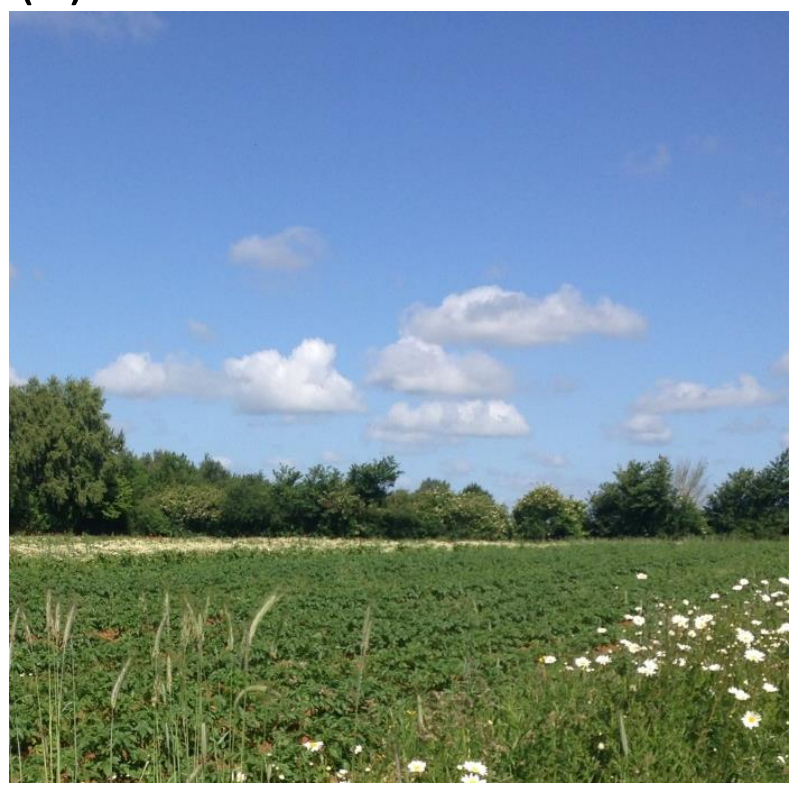

(d) $H A \quad A B \quad A D \quad H S \quad C C$ EB PN $D C$ PF $A P$

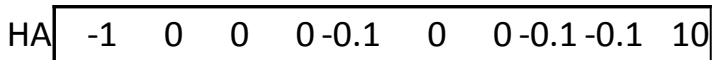

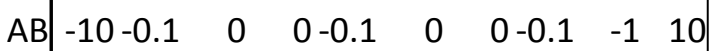

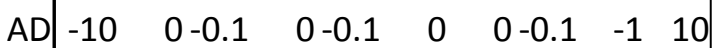

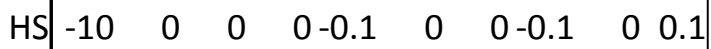

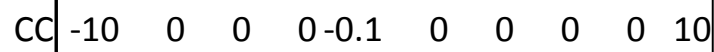

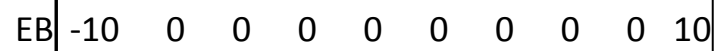

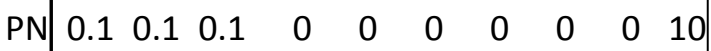

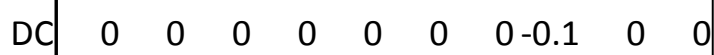
PF-0.1 $00 \begin{array}{ccccccc}0 & 0 & 0-0.1 & 0 & 0-0.1 & 0 & 0\end{array}$

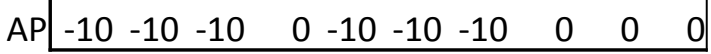




\section{Online Appendix:}

707

708

709

710

711

712

713

714

715

716

717

718

719

720

721

722

723

724

725

726

727

Appendix A: A trait-based assembly model.

We consider a generalised version of the Loeuille and Loreau (2005) model developed by Brännström et al. (2011) which depicts the per capita population change rate as a function of population growth derived from prey consumption minus mortality from senescence, predation and interference competition:

$$
\frac{1}{n_{i}} \frac{d n_{i}}{d t}=\lambda \sum_{j=0}^{s} \frac{P_{j i} n_{j}}{\exp \left(\Delta r_{i j}\right)}-\left(D_{i}+\sum_{j=1}^{s} P_{i j} n_{j}\right)-\sum_{j=1}^{s} C_{i j} n_{j}
$$

where $\lambda$ is the conversion efficiency for the consumptive interactions. The per-capita predation rate of species $i$ preying on species $j$ is set to be trait-mediated, $P_{j i}=\gamma_{0} N\left(\Delta r_{i j}-\mu, \sigma_{\gamma}\right)$, where $N(a, b)$ is the probability density function of normal distribution with mean $a$ and standard deviation $b, \Delta r_{i j}$ the trait difference of species $i$ and $j, r_{i}-r_{j}$ (considering the logarithm of body size relative to that of the autotroph). The coefficients $\mu$ and $\sigma_{\gamma}$ represent the optimal trait ratio of predator to prey and the dietary breadth of the predator. The natural mortality is also assumed to be trait-mediated, $D_{i}=d_{0} \exp \left(-r_{i} / 4\right)$ (Peters, 1983). The intensity of interference competition is at its maximum when the two competing species have identical traits, $C_{i j}=k_{0} N\left(\Delta r_{i j}, \sigma_{k}\right)$. In addition, the dynamics of the autotroph (resource base) is governed by, $d n_{0} / d t=n_{0}\left(g-k_{0} n_{0}-\sum_{j=1}^{s} P_{0 j} n_{j}\right)$, where $g$ is the intrinsic population growth rate (see detail model description in Brännström et al. 2011). The resources $n_{0}$ are disturbed by adding a periodic perturbation (sine form with pulse 10 and magnitude 100). 